\title{
Efficacy of SGLT2 inhibitors in glycemic control, weight loss and blood pressure reduction: a systematic review and meta-analysis
}

\author{
Lana Catani Pinto, Dimitris Varvaki Rados, Luciana Reck Remonti', Caroline Kaercher Kramer, \\ Cristiane Bauermann Leitao, Jorge Luiz Gross
}

From 20th Brazilian Diabetes Society Congress

Porto Alegre, Brazil. 11-18 November 2015

\section{Background}

Sodium-glucose cotransporter 2 inhibitors (SGLT2i) are a novel antidiabetic class that inhibits glucose reabsorption and produce glycosuria. These medications are being increasingly used as dual therapy with metformin for type 2 diabetes (T2D) treatment, due to their beneficial effect on weight and blood pressure. Three agents are approved for clinical use and they may differ on potency due to inhibition of only renal or both renal and bowel glucose transportation.

\section{Objective}

to evaluate the efficacy of SGLT2i, dapagliflozin (DAPA), canagliflozin (CANA) and empagliflozin (EMPA), on HbA1c, weight and blood pressure (BP) in comparison with placebo and other antidiabetic medications.

\section{Materials and methods}

MEDLINE, Cochrane central and EMBASE databases were searched for randomized clinical trials (RCTs) including patients with T2D allocated to SGLT2i for at least 12 weeks. A direct and network meta-analysis (NMA) were conducted.

\section{Results}

Thirty-nine RCTs were included (25.468 patients). CANA $300 \mathrm{mg}$, EMPA $25 \mathrm{mg}$ and DAPA $10 \mathrm{mg}$ were associated with better g.lycemic control (HbA1c $-1.01 \%,-0.69 \%$, $-0.51 \%$, respectively), and weight loss $(-2.66 \mathrm{~kg} ;-1.81 \mathrm{~kg}$; $-1.80 \mathrm{~kg}$, respectively) when compared to placebo. In NMA, CANA $300 \mathrm{mg}$ was superior to the others SGLT2i

\footnotetext{
* Correspondence: lucianalreck@gmail.com

Hospital de Clínicas de Porto Alegre, Porto Alegre, Brazil
}

for HbA1c (EMPA $25 \mathrm{mg}$ : $0.22 \%$ and DAPA $10 \mathrm{mg}$ : $-0.26 \%$ ), and weight (EMPA $25 \mathrm{mg}:-1.06 \mathrm{~kg}$ and DAPA $10 \mathrm{mg}:-0.84 \mathrm{~kg})$. CANA $300 \mathrm{mg}$ and DAPA $10 \mathrm{mg}$ decreased systolic BP $(-4.77 \mathrm{mmHg}$ and $-2.66 \mathrm{mmHg}$, respectively) and diastolic $\mathrm{BP}(-1.99 \mathrm{mmHg}$ and $-1.76 \mathrm{mmHg}$, respectively) in comparison to placebo. SGLT2i were similar to metformin and sulphonylurea regarding to $\mathrm{HbA} 1 \mathrm{c}$, but superior to DPP4 inhibitors $(-0.15 \%)$ (Figure 1). Furthermore, SGLT2i were better than metformin, sulphonylurea, and DPP4 inhibitors for reduction of weight $(-1.04 \mathrm{~kg},-4.76 \mathrm{~kg}$ and $-2.45 \mathrm{~kg}$, respectively) and systolic BP $(-5.86 \mathrm{mmHg},-5.44 \mathrm{mmHg}$ e $-4.43 \mathrm{mmHg}$, respectively). SGLT2i were also better than sulphonylurea and DPP4 inhibitors for diastolic BP lowering (-2.59 $\mathrm{mmHg},-1.89 \mathrm{mmHg}$, respectively). Initial combination of SGLT2i plus metformin resulted in greater reduction of $\mathrm{HbA} 1 \mathrm{c}$ than SGLT2 plus DPP4i $(-0.53 \%$ vs. $-0.19 \%)$.

\section{Conclusion}

In T2D patients, SGLT2i were superior to placebo for all outcomes analyzed, and CANA seems to be the most potent among then. SGLT2i are as effective as metformin and sulphonylurea and superior to DPP4 inhibitors for HbA1c, but more potent than these classes regarding weight and BP reduction.

Published: 11 November 2015

doi:10.1186/1758-5996-7-S1-A58

Cite this article as: Pinto et al.: Efficacy of SGLT2 inhibitors in glycemic control, weight loss and blood pressure reduction: a systematic review and meta-analysis. Diabetology \& Metabolic Syndrome 2015 7(Suppl 1):A58. 


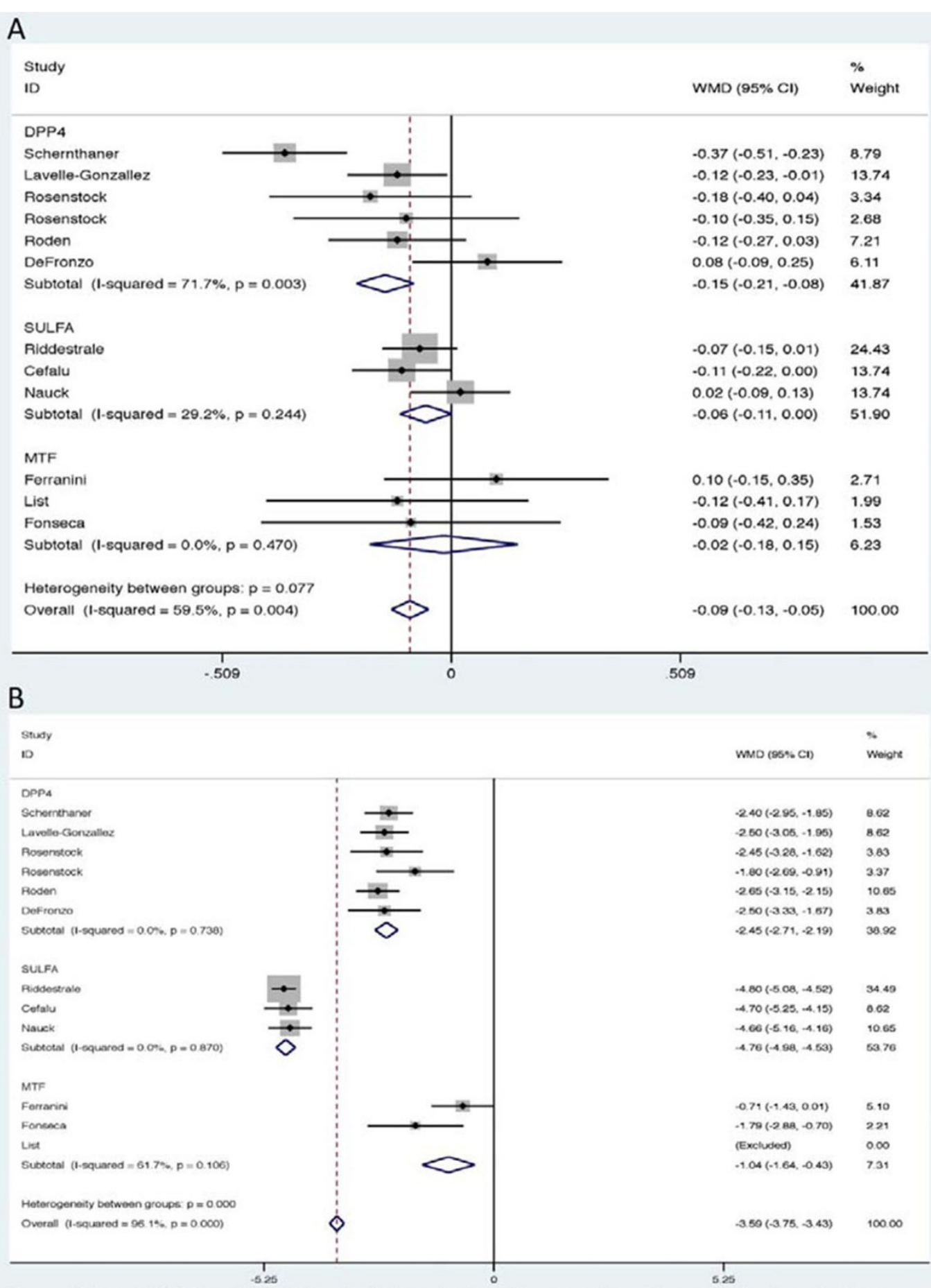

Figure. Effects of SGLT2i on HbA1C (A) and weight reduction (B) compared to other antidiabetic medications

Figure 1 Effects of SGLT2i on HbA1c (A) and weight reducAon (B) compared to other antidiabetic medications 